\title{
Video Coding with Motion Estimation at the Decoder
}

Claudia Tonoli, Pierangelo Migliorati, and Riccardo Leonardi

\section{Introduction}

Compression efficiency is a fundamental requirement in all video coding systems. 5 It becomes even more important in the case of small wireless devices because they 6 are often subject to tighter power and bandwith constraint. In predictive video cod- 7 ing schemes, the key feature to achieve compression efficiency is motion estimation. 8 The basic idea of this approach is to exploit the temporal redundancy across frames, 9 estimated using the motion information. Usually motion estimation is performed at 10 the encoder side, and then the motion field is transmitted to the decoder, together 11 with the compressed prediction error. The decoding of each block of a frame consists 12 in simply extracting the predictor, which is identified thanks to the motion vector, 13 from the reference frame and adding the prediction residue. Both the motion vector 14 and the residue are computed by the encoder. The decoding process, that is applied 15 blockwise, cannot prescind from their complete transmission.

Despite that such systems are based on a blockwise decoding, it is not true that 17 each block of a frame is independent from its neighbors. On the contrary, the struc- 18 ture of natural images generally imposes a strong spatial correlation among adjacent 19 blocks, but such spatial correlation is not completely exploited in traditional system. 20 A coding technique, able to reduce the redundancy between the already decoded 21 part of the frame and the motion information, would improve the compression effi- 22 ciency, possibly completely discarding the motion information from the transmitted 23 bitstream.

Thanks to arithmetic coding and prediction techniques, motion information is 25 nowadays compressed very efficiently. Nevertheless, especially for low bit rate video 26 coding, the motion field still has a nonnegligible impact on the overall bit-rate. The 27 idea of skipping the transmission of the motion information and reestimate it at the 28 decoder has recently attracted an increasing interest. For example, in [1] an algorithm 29 for motion derivation at the decoder side for the H.264/AVC codec is presented. 30 This algorithm is based on a template similar to those used in texture coding.

C. Tonoli (殴, P. Migliorati, and R. Leonardi

Department of Electronics for Automation, Signals and Communication Lab, University

of Brescia, Brescia, Italy

e-mail: \{name.surname\}@ing.unibs.it 
In this chapter, we propose a method for motion estimation at the decoder. The 32 proposed approach relies on the knowledge of the prediction residue, transmitted by 33 the encoder, and it is based on Least Square Error prediction. Preliminary simulation 34 results seem to be very promising.

The chapter is structured as follows. In Sect. 2, a brief description of the use of 36 motion compensation in predictive video coding schemes is given. The proposed 37 algorithm is described in detail in Sect. 3, and simulation results are presented and 38 discussed in Sect. 4. Concluding remarks are given in Sect. 5.

\section{Motion Compensation in Traditional Video Coding Schemes}

Predictive video coding is based on motion estimation at the encoder and motion 41 compensation at the decoder. In this section, the basic ideas of predictive video 42 coding are briefly introduced. The highlighted details will be useful in the following. 43 For a more complete description of this topics, we refer the reader to [2-4]. 44

In predictive coding, the suitable predictor for each block is determined at the en- 45 coder, usually performing a block based motion estimation. The prediction residue, 46 i.e., the difference between the current block and its predictor, is computed and en- 47 coded. The information sent to the decoder includes the residue, together with the 48 motion field.

The decoder reconstructs each frame operating in a strictly blockwise mode, 50 since each block is reconstructed independently from its neighbors. The motion 51 vector associated to the current block is used as an index for the set of possible 52 predictors. Once the correct predictor has been identified, the prediction residue is 53 decoded and added to the prediction values.

This method obviously requires that one motion vector is transmitted for each 55 block. Due to the efficiency of modern entropy coding techniques, the transmission 56 of the motion field is in general not very expensive in terms of bit-rate. Especially 57 in high bit-rate coding, where DCT coefficients quantization is very fine, motion 58 information represents a small part of the overall transmitted rate. Nevertheless, in 59 low bit-rate coding the amount of rate assigned to the signal coefficient is lower, so 60 the motion rate becomes much more important.

\section{The Proposed Algorithm for Motion Estimation at the Decoder}

In this section, the proposed method of motion estimation at the decoder side is 64 presented.

First of all, the fundamental ideas are introduced, focusing on the definition of 66 the side information. The algorithm is then outlined, sketching a structure that can 67 be applied with different spatial coherence evaluation parameters. Finally, the LSE 68 based parameter is introduced, and its computation is described in some detail. 


\subsection{Decoder Side Information}

The term "Side Information" can be found very frequently in recent papers on video 71 coding, and it often acquires different meanings, depending on which specific field 72 we are looking at. To a very general extent, it refers to pieces of information that 73 are not exactly the values of the coded signal, but a somewhat higher level corre- 74 lated information, which is indeed crucial for the proper decoding of the signal. In 75 particular, the centrality of the concept of side information and the way the side 76 information is dealt with is one of the distinguishing elements of the Distributed 77 Video Coding (DVC) paradigm (see, for example, [5,6]). In this paradigm, each 78 frame is encoded independently from the others. Due to such assumption of inde- 79 pendent frame coding, the motion estimation is not performed at the decoder, and 80 the motion field has to be inferred at the decoder side, based on the side information. 81

Borrowing the concept of side information from Distributed Video Coding, in 82 this work, we assume that the side information for the current frame corresponds 83 to the previously decoded frames. In more detail, since the encoder motion com- 84 pensation algorithm is known, when the decoder begins to decode a frame block, it 85 already has some knowledge about that block, in terms of correlated information. 86 It is known for sure that the motion compensated predictor for that block belongs 87 to the block matching reference frame, and, more precisely, to the search window. 88 In fact, the motion vector in motion compensation behaves exactly as an index for 89 the set of predictors corresponding to the search window. Since the reference frame 90 has already been decoded, the set of candidate predictors for the current block is 91 completely known. Equivalently, it is possible to say that the final reconstructed 92 block will be one of these candidate predictors corrected with the received predic- 93 tion residue for that block.

Moreover, we introduce an a priori hypothesis that, despite its generality, turns 95 out to be true in the great majority of cases. We assume that the signal to be coded 96 is characterized by "spatial continuity," i.e., edges preserve their continuity across 97 the block boundaries. This means that, given the neighborhood of a block, it is pos- 98 sible to infer that the more suitable predictor in a candidate set will be the one that 99 matches at best the neighborhood edges. See Fig. 1 for an example of a well matched 100 and a bad matched predictor, respectively. If we assume that block decoding is per- 101 formed in raster scan order, the causal neighbors of the current block have already 102 been decoded. Therefore, it is possible to use the information carried out by the 103 position of their edges to try to match the candidate predictors.

These remarks about the side information role will be the basis for the selection 105 of a predictor for motion compensation in the absence of the motion vector, and for 106 the consequent motion estimation at the decoder.

\subsection{Outline of the Predictor Selection Algorithm}

Let us consider a predictive coding scheme based on motion compensation, as 109 described in Sect. 2. Our aim is to avoid the transmission of motion vectors, 110 

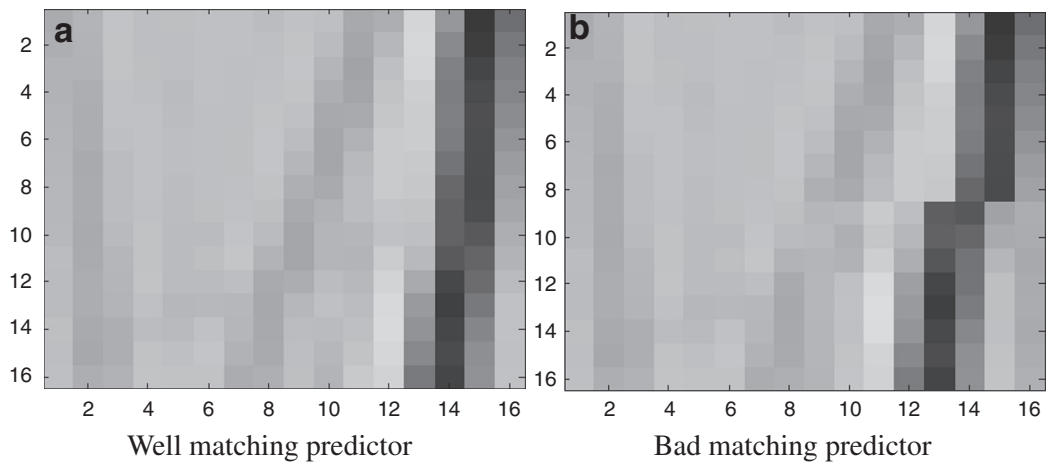

Fig. 1 Spatial continuity at block edges. (a) Well matching predictor. (b) Bad matching predictor
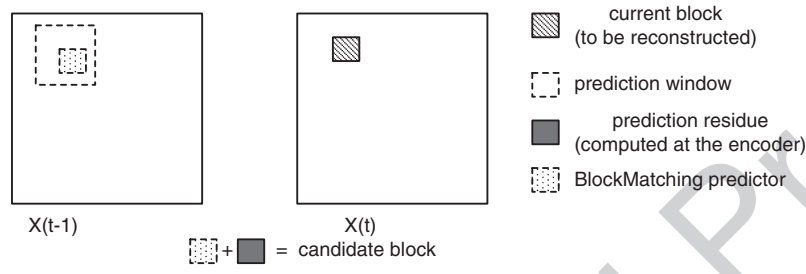

Fig. 2 Candidate set generation

nevertheless achieving a reconstruction quality close to that obtainable in case of 111 transmission of the whole motion field. In order to try to do that, we apply the prin- 112 ciples about side information described in Sect. 3.1.

For each block to be reconstructed, the set of candidate predictors is generated, as 114 depicted in Fig. 2. A ranking of the candidates is then performed, in order to find out 115 which candidates fits at best the coherence conditions, as described in the following 116 lines. The already decoded causal neighborhood of the current block is considered. 117 The macroblock composed by the current block and its three causal neighbors is 118 constructed, replacing the current block with the tested candidate. A parameter $p 119$ measuring the matching of the candidate block with the neighborhood is computed, 120 in order to select the predictor that guarantees the best matching with the side in- 121 formation. Obviously, the matching parameter plays a crucial role in the algorithm 122 performance, since it has to capture the matching of each candidate predictor and 123 to select the most suitable one. In this chapter, we present a method based on Least 124 Squared Error prediction. Such method will be described in more detail in Sect. 3.3. 125 The reason why LSE prediction has been chosen to highlight the spatial coherence 126 is that such technique is based itself on the exploitation of the correlation among ad- 127 jacent pixels. The presented algorithm relies on the principle that a block correlated 128 to the given neighborhood should be well predictable from the neighborhood, while 129

AQ2 a less correlated block should produce a greater prediction error (Fig. 3).

Since we want to control at the encoder side the quality of the reconstructed 131 signal, as it usually happens in predictive coding schemes, we apply our method 132 
Fig. 3 Scheme of the proposed system

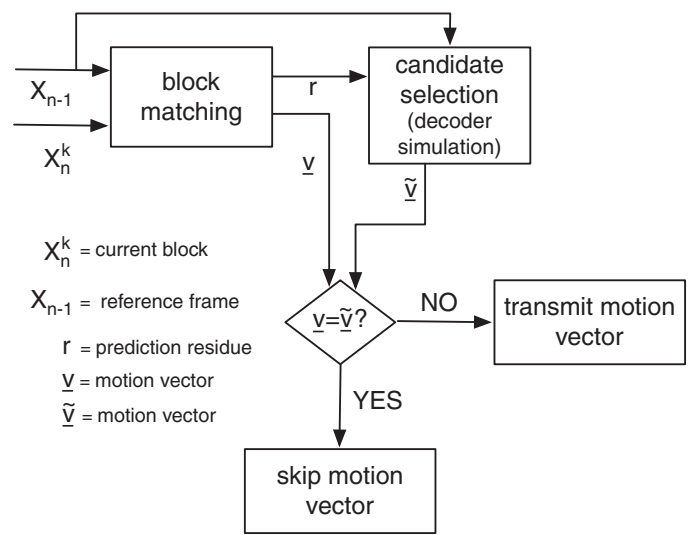

Define the criteria for spatial consistence and define a

$\forall$ block in parameter $p$

1. generate the candidate set:

a. extract all the block belonging to the block matching

window of the reference frame

b. add the prediction residue to each extracted block

$\forall$ block in the candidate set:

a. compute the consistence parameter $p$
3. select the candidate that maximizes/minimizes

Fig. 4 Algorithm structure

first at the encoder. In detail, for each block, the encoder simulates the operations of 133 the decoder, and, based on the quality of the reconstructed block, decides whether 134 the motion vector for that block is omissible or not. In our implementation, a sim- 135 ple threshold on the quality of the reconstructed block has been applied. A more pre- 136 cise rate-distortion analysis, like the one performed for example in the H.264/AVC 137 encoder, could lead to a performance improvement because the effect of a motion 138 vector skip on the overall reconstructed PSNR could be estimated more precisely. 139

\subsection{Candidate Selection Based on Least Square Error Prediction}

In the framework described in Sect. 3.2, in the absence of the motion information, 141 the only criterion for the decoder to select one block among the candidates is the 142 good match with the intra side information, i.e., the neighborhood.

The decoder-based motion compensation algorithm has been implemented ac- 144 cording to the steps described in Fig. 4. As stated in step 2, each candidate needs to 145 be tested in order to produce the parameter $p$, i.e., a "measure" of the correlation 146 of that block with the known neighbors, and to get a ranking of the candidates. The 147 steps to be performed to obtain such ranking are listed below. 
1. Test each candidate block in the following way:

(a) Prediction of the upper left quadrant; for each pixel, the correlation matrix 150 is reestimated, based on the neighbors and on the true value of the past 151 pixels in the block (i.e., the predicted pixels in the past are not considered 152 in the estimation)

(b) Compute the MSE on the upper left quadrant between the predicted block 154 and the true candidate block

2. Choose the candidate that results to be more predictible, i.e., such that the MSE 156 computed at the step 1 is smaller than that obtained for any other candidate $\quad 157$ $(p=\mathrm{MSE})$.

The LSE prediction has been implemented as described in [7], where it is used to 159 perform still image compression: each pixel is predicted, based on its causal neigh- 160 borhood, and the prediction residue is encoded and transmitted. The prediction is 161 shown to be orientation adaptive.

The LSE prediction computation is now briefly reported. Further details are given 163 in [7]. For each pixel, a training window is set, as depicted in Fig. 5. According to the 164 training values, the prediction coefficients are adaptively computed. The correlation 165 matrix is estimated as described in the following.

$$
c_{i}=\left[x_{i-1}, x_{i-2}, \ldots, x_{i-L}\right]
$$

where $x_{i-j}$ is the $j$ th causal neighbor of $x_{i}$, for $i=1,2, \ldots, L$.

A $W \times L$ matrix, whose rows are the $c_{i}$ vectors, is created:

$$
C=\left[\begin{array}{c}
c_{1} \\
c_{2} \\
\vdots \\
c_{W}
\end{array}\right]
$$

The covariance matrix is then computed as:

$$
R_{x x}=C^{T} C
$$

while the covariance vector $r_{x}$ is computed as

$$
r_{x}=C^{T} y
$$

Fig. 5 Sliding training window

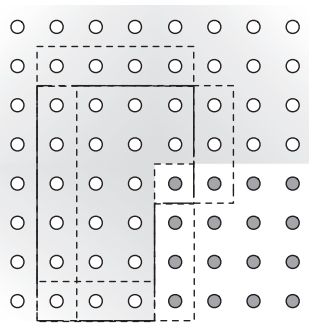

correct past neighbors --- training windows ○ candidate block pixels 
where

$$
y=\left[x_{n-1}, \ldots, x_{n-L}\right]^{T}
$$

According to the theory of least squared error prediction, the coefficient vector $a 172$ is computed as

$$
a=\left(C^{T} C\right)^{-1}\left(C^{T} y\right)
$$

The main drawback of this algorithm is its huge computational complexity, 174 due to the frequent matrix inversion operations that are needed. In the litera- 175 ture, several techniques have been presented to reduce the complexity [8]. In our 176 implementation, the edge based technique presented in [7] has been used. It can be 177 seen that the complexity can be reduced with a performance impairment of about 178 $1 \%$ more wrong block.

\section{Experimental Results}

In this section, the performance of the proposed algorithm is presented and 181 discussed.

In order to evaluate the proposed method, the percentage of correctly recon- 183 structed motion vectors has been computed. As a groundtruth reference, the lossless 184 case is considered. On the original CIF format sequence, the block matching is per- 185 formed, on blocks of size $16 \times 16$, as a means to compute the motion field and the 186 prediction residue. Since the work presented in this paper is aimed at exploring a 187 new field, many optimizations have not been introduced yet: no multiple reference 188 is considered for the block matching, and the reference for each frame is the previ- 189 ous frame.

When the prediction residue has been computed, the motion estimation method 191 is applied and the percentage of correctly estimated motion vectors is computed for 192 each frame. It is worth to remark that no error propagation is taken into account in 193 the presented results. It is always assumed that the encoder controls the decoding 194 process and, when a block cannot be correctly estimated in the absence of motion 195 vector, the motion information is transmitted. So the neighbors of a block are always 196 correct, either because their motion vector has been estimated correctly or because 197 motion has been transmitted.

In Table 1, the results in terms of percentage of correct blocks is reported for 199 the first two frames of four test sequences, namely Foreman, Mobile, Highway, 200

t1.1 Table 1 Percentage of correctly predicted blocks in the lossless case

\begin{tabular}{lll}
\cline { 2 - 2 } t1.2 & Sequence & Correctly estimated motion vectors $(\%)$ \\
t1.3 & Foreman & 75.93 \\
t1.4 & Mobile & 34.67 \\
t1.5 & Highway & 84.92 \\
t1.6 & Harbour & 41.22
\end{tabular}


and Harbour. It can be noticed that the performance is strongly dependent on the 201 sequence content. For Highway and Foreman, very good results can be achieved, 202 whereas for Mobile and Harbour, the algorithm is less effective.

In order to give an idea of how the presented algorithm could perform in a real- 204 istic scenario, it has been applied to lossy coding. In particular, low bit rate coding 205 has been considered because in this case, skipping the motion information could be 206 particularly advantageous.

In more detail, the rate and PSNR values for the case of transmission of the whole 208 motion field have been obtained using a simplified H.264 codec. The block size 209 has been set to 16 and the considered prediction mode is P, i.e., mono-directional 210 prediction, with a single reference picture. No deblocking has been performed on the 211 reconstructed frame. An important remark has to be given about the rate estimation. 212 The coding efficiency in modern predictive codec, such as H.264 codec, depends 213 heavily on how arithmetic coding is performed. Since our method has not been really 214 implemented in H.264 yet, it is impossible to measure exactly the rate savings. In 215 order to produce a reliable estimate, the bits devoted to the motion transmission for 216 each block have been computed, and, for the correctly predicted block, the result 217 has been subtracted from the overall bit-rate. A signalling overhead has also been 218 taken into account.

The estimated performance of the considered method is reported, for the first ten 220 inter-frames (i.e., frames from 2 to 11 , since the first frame is intra-coded) of the 221 Foreman and Harbour sequence in CIF format, at $15 \mathrm{fps}$, in Fig. 6a and b.

In order to help a more precise performance assessment, the percentage of 223 skipped motion vector is also reported, in Table 2. In the case of lossy coding, it 224 can happen that the selected motion vector is not the correct one, but the selected 225 candidate is not too different from the correct block. In this case, it can be seen that 226 the proposed method can lead to a slight performance improvement.

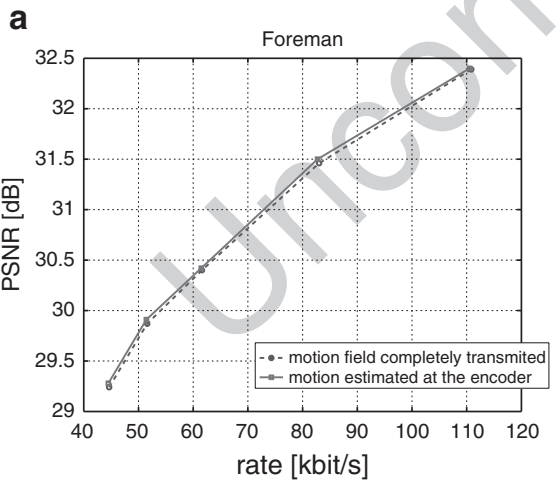

PSNR curves for the Foreman sequence

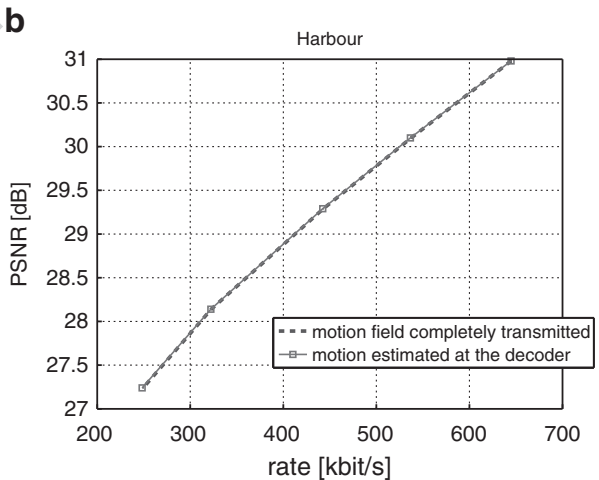

PSNR curves for the Harbour sequence.

Fig. 6 PSNR curves for the test sequences Foreman and Harbour. (a) PSNR curves for the Foreman sequence. (b) PSNR curves for the Harbour sequence 
t2.1 Table 2 Percentage of correctly predicted blocks for the lossy compression of the Foreman sequence

\begin{tabular}{lll}
\cline { 2 - 3 } t2.2 & \multicolumn{1}{c}{$\begin{array}{l}\text { Percentage of skipped } \\
\text { motion vectors }\end{array}$} \\
t2.3 & PSNR & 21.03 \\
t2.4 & 31.51 & 15.0 \\
t2.5 & 30.42 & 14.69 \\
t2.6 & 29.91 & 17.50 \\
t2.7 & 29.28 &
\end{tabular}

\section{Conclusions}

Compression efficiency is particularly important in small, low power devices. Side 229 information at the decoder side, i.e., correlated information about the signal that 230 has to be decoded, can be exploited to improve compression efficiency in predictive 231 video coding. Starting from the side information, the encoder can infer important 232 knowledge that helps in decoding the signal. As an example of how side informa- 233 tion at the decoder side can be exploited in video coding, in this chapter, we have 234 proposed a method that partially avoids the transmission of motion vectors in pre- 235 dictive video coding schemes based on motion compensation. Simulation results 236 show that the proposed approach can lead to bit-rate savings.

\section{References}

1. Kamp S, Evertz M, Wien M (2008) Decoder side motion vector derivation for inter frame video 239 coding. In: Proceedings of 15th IEEE international conference on image processing 2008 (ICIP 240 2008), 12-15 Oct 2008, pp 1120-1123 241

2. Wiegand T, Sullivan G, Bjontegaard, G (2003) Overview of the H.264/AVC video coding stan- 242 dard. IEEE Trans Circuits Syst Video Technol 13(7):560-576 243

AQ3 3. Wiegand T, Sullivan G, Luthra A (2003) Draft ITU-T recommendation and final draft 244 international standard of joint video specification $\quad 245$

4. Kappagantula S, Rao KR (1985) Motion compensated interframe image prediction. IEEE Trans 246 Commun 33(9):1011-1015 247

5. Aaron A, Zhang R, Girod B (2002) Wyner-Ziv coding for motion video. In: Proceedings of 248 36th Asilomar conference on signal, systems and computers (ACSSC '02), Pacific Grove, CA, 249 vol 1, pp 240-244 250

6. Puri R, Ramchandran K (2003) PRISM: a new reversed multimedia coding paradigm. In: Pro- 251 ceedings of the IEEE internatinal conference on image processing, Barcelona, Spain, Sept 2003252

7. Li X, Orchard MT (2001) Edge directed prediction for lossless compression of natural images. 253 IEEE Trans Image Process 10(6):813-817 254

8. Li X, Orchard MT (2002) Novel sequential error-concealment techniques using orientation 255 adaptive interpolation. IEEE Trans Circuits Syst Video Technol 12(10):857-864 256

AQ4 9. Wu X, Barthel K (1998) Piecewise 2D autoregression for predictive image coding. In: Proceed- 257 ings of the IEEE international conference on image processing, Chicago, IL, Oct 1998, vol 3, 258 pp 901-904

10. Park JW, Kim JGK, Lee SU (1997) DCT coefficients recovery-based error concealment tech- 260 nique and its application to the MPEG-2 bit stream error. IEEE Trans Circuits Syst Video 261 Technol 7(6):845-854 